\title{
Uniportal video-assisted thoracic surgery Roman experience-a report of the first 16-month Roman experience
}

\author{
Dania Nachira ${ }^{1}$, Elisa Meacci ${ }^{1}$, Leonardo Petracca Ciavarella ${ }^{1}$, Marco Chiappetta ${ }^{1}$, Giulia De Santis ${ }^{1}$, Gian \\ Maria Ferretti ${ }^{1}$, Maria Giovanna Mastromarino ${ }^{1}$, Venanzio Porziella ${ }^{1}$, Maria Letizia Vita ${ }^{1}$, Maria Teresa \\ Congedo $^{1}$, Alfredo Cesario ${ }^{1}$, Mahmoud Ismail ${ }^{2}$, Diego Gonzalez-Rivas ${ }^{3,4}$, Stefano Margaritora ${ }^{1}$ \\ ${ }^{1}$ Department of General Thoracic Surgery, Catholic University of Sacred Heart, Fondazione Policlinico Universitario “A.Gemelli”, Rome, Italy; \\ ${ }^{2}$ Competence Center of Thoracic Surgery, Department of Surgery, Charité-Universitätsmedizin Berlin, Berlin, Germany; ${ }^{3}$ Department of Thoracic \\ Surgery, Shanghai Pulmonary Hospital, Tongji University School of Medicine, Shanghai 200433, China; ${ }^{4}$ Department of Thoracic Surgery and \\ Minimally Invasive Thoracic Surgery Unit (UCTMI), Coruña University Hospital, Coruña, Spain \\ Contributions: (I) Conception and design: D Nachira, E Meacci, M Ismail, D Gonzalez-Rivas; (II) Administrative support: S Margaritora, A Cesario; \\ (III) Provision of study materials or patients: V Porziella, ML Vita, MT Congedo; (IV) Collection and assembly of data: G De Santis, M Chiappetta, \\ L Petracca Ciavarella, GM Ferretti, MG Mastromarino; (V) Data analysis and interpretation: D Nachira, E Meacci; (VI) Manuscript writing: All \\ authors; (VII) Final approval of manuscript: All authors. \\ Correspondence to: Dania Nachira, MD. Department of General Thoracic Surgery, Fondazione Policlinico Universitario “A.Gemelli”, Largo A. \\ Gemelli, 8, 00168 Rome, Italy. Email: danynac@libero.it.
}

Background: The acceptance of uniportal video-assisted thoracic surgery (U-VATS) for thoracic procedures has been growing worldwide. This study reports one of the widest Italian U-VATS experiences.

Methods: The prospectively collected data of 237 patients underwent a U-VATS procedure, between May 2016 and September 2017, were retrospectively reviewed. A wide range of procedures, like major and minor lung resections, esophageal surgery, pleural and mediastinal one, was performed. The main aim of the study was evaluating general outcomes in terms of safety and effectiveness, and analyzing short-term results of U-VATS approach.

Results: The mean age of population was $59.93 \pm 16.03$ years. In 208 cases $(85.3 \%)$ a U-VATS lung resection was performed, in 10 cases $(4.1 \%)$ an esophagectomy or an esophageal diverticulectomy, in $15(6.1 \%)$ a mediastinal procedure and in $11(4.5 \%)$ a toilette for pleural empyema or removal of pleural lesions. The chest tube duration was $4.24 \pm 3.73$ days and the postoperative hospital stay was $4.62 \pm 4.59$ days. The intraoperative and thirty-day mortality were null. Mean level of pain in I postoperative day was $2.30 \pm 1.26$ on VAS scale and the mean duration was of $1.54 \pm 1.21$ days. In $93 \%$ of cases there was a resolution of pain after chest tube removal. Furthermore, the average level of cosmetic satisfaction was $2.73 \pm 0.49$ (measured on a $0-3$ scale).

Conclusions: According to our experience, U-VATS seems to be a safe and practicable mini-invasive technique, above all for surgeons who already have thoracoscopy experience or made proper training attending multilevel courses, hands-on conferences and wet-labs.

Keywords: Uniportal video-assisted thoracic surgery (U-VATS); lung cancer; lung major resections; mediastinal surgery

Submitted Jan 28, 2018. Accepted for publication Mar 13, 2018.

doi: $10.21037 /$ jtd.2018.03.119

View this article at: http://dx.doi.org/10.21037/jtd.2018.03.119 


\section{Introduction}

Since its introduction in 1998 (1), uniportal video assisted thoracic surgery (U-VATS) has been spreading and gaining success among thoracic surgeons. But it was after GonzalesRivas's first reports (2-6) of major pulmonary resections and complex procedures (like bronchoplasties) that uniportal VATS has started to show its great potential of safe and feasible technique able to change the scenario of Thoracic Surgery worldwide.

In the last few years, hundreds of dedicated courses and master classes have been organized and successfully attended from Europe to Asia (7-9). So, in a lot of centers like ours, U-VATS has become the favorite approach for the treatment of a lot of chest diseases, not only lung cancer.

In the present article we report our experience with U-VATS technique since the beginning in May 2016, and nowadays one of the largest U-VATS series in Italy, evaluating general outcomes in terms of safety and effectiveness, and analyzing short-term results of U-VATS approach for a wide range of procedures (minor and major lung surgery, esophageal surgery, mediastinal one etc.).

\section{Methods}

Between May 2016 and September 2017, 244 U-VATS procedures (on 237 patients) were performed at General Thoracic Surgery Department, Fondazione Policlinico Universitario “A. Gemelli" in Rome (Italy). The prospectively collected clinical data of patients underwent these surgeries were retrospectively reviewed.

All patients provided written informed consent before operation and underwent preoperative evaluation, including: routine blood tests, electrocardiography, radiological examinations [chest/total body computed tomography (CT), positron emission tomography (PET)-CT...] and pulmonary function test when necessary.

Postoperative pain was evaluated by Visual Analog Scale (VAS scale) in I postoperative day and after chest tube removal. The duration of pain, the types of painkillers administered, the incidence of paresthesia 7 days after the operation and cosmetic results (on a scale from 0 to 3 ) were also recorded.

\section{Surgical technique}

All procedures were performed by the same surgical team, under general anesthesia and single-lung ventilation. The

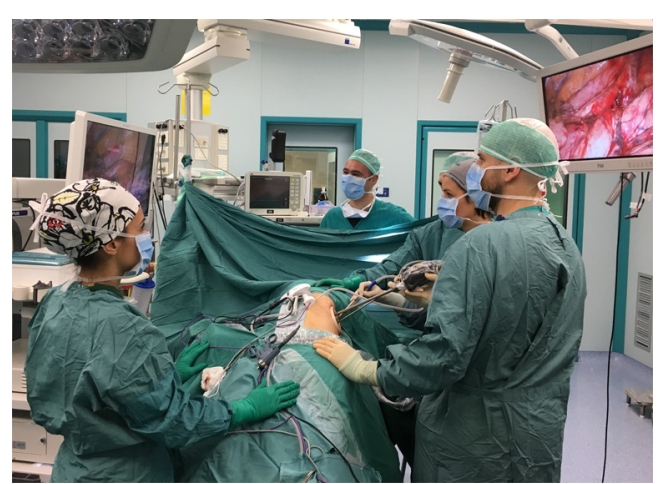

Figure 1 Position of the patient, of the surgical equipe and scrub nurse during U-VATS procedure. U-VATS, uniportal videoassisted thoracic surgery.

patients were placed in lateral decubitus (Figure 1) with their arms flexed and stretched towards their head (10). The single $3-4 \mathrm{~cm}$ muscle-sparing incision was made on the midaxillary line in the IV or V intercostal space, depending on the location of the lesion. For managing lesions localized in the upper lobes or centrally, a IV space could be favourite. A wound protector was placed and a $10 \mathrm{~mm} 30^{\circ}$ thoracoscope and endoscopic instruments were all introduced through the same incision, with the camera always held in the upper part of the incision. The width of the incision together with the good mobilization of the lung obtainable with the curved shaped, dual pivot instruments allowed a good palpation of lung parenchyma for localizing nodules.

Endostaplers and energy dissectors were used for dissecting and cutting lung parenchyma and vascular structures. The same principles for oncological radicality were observed as in open surgery. A complete lymphadenectomy was performed in case of primary lung cancer.

The specimen was removed by an Endobag. At the end of each procedure, an extrapleural paravertebral intercostal nerve block was performed (Figure 2), infiltrating $3 \mathrm{~mL}$ of ropivacaine $(4.75 \mathrm{mg} / \mathrm{mL})$ in $3-4$ intercostal spaces above and below the incision, under endoscopic view (10). Usually only one chest drain ( 24 or $28 \mathrm{Fr}$ ) was placed at the end of the operation, through the same incision and in its upper part (Figure 3); two drains were placed only in case of pleural toilette for empyema or when required.

\section{Postoperative management}

All patients underwent a good thoracic analgesia (local nerve block during the operation and systemic administration 


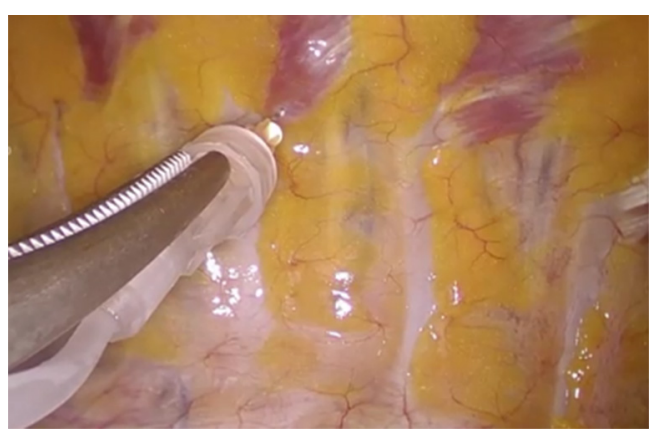

Figure 2 Intercostal nerve blockage under endoscopic view.

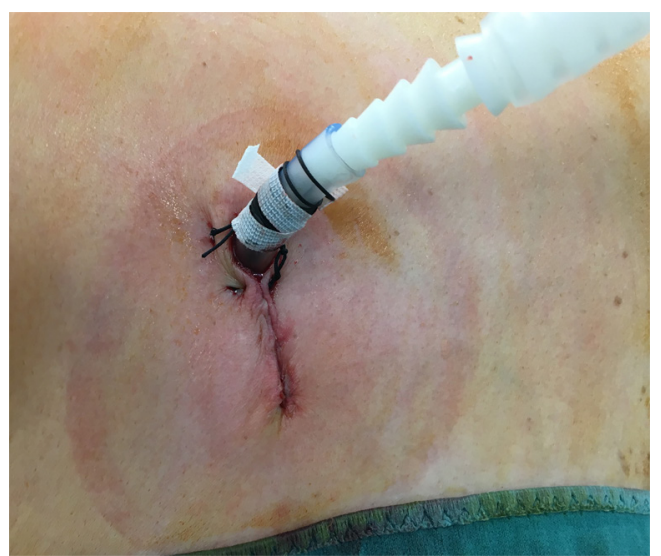

Figure 3 Position of the chest tube in the U-VATS incision. U-VATS, uniportal video-assisted thoracic surgery.

Table 1 Demographical and clinical characteristics of the patients

\begin{tabular}{lc}
\hline Characteristics & $\mathrm{N}=237$ \\
\hline Age (years) & $59.93 \pm 16.03$ \\
Gender (male/female) & $52(21.9 \%)$ \\
Smoking & $82(34.6 \%)$ \\
Ex-smoking history & $25.94 \pm 4.07$ \\
BMI & $70(29.5 \%)$ \\
COPD & $49(20.7 \%)$ \\
Heart disease & $106(44.7 \%)$ \\
Arterial hypertension & $21(8.8 \%)$ \\
Diabetes & 15 (6.3\%)/174 (73.4\%)/48 (20.2\%) \\
ASA score: $1 / 2$ /3 & $117(49.4 \%)$ \\
Previous neoplasia & \\
\hline BMl, body mass index; COPD, chronic obstructive pulmonary \\
disease; ASA Score, American Society of Anesthesiologists \\
Score.
\end{tabular}

of painkillers), forced mobilization and respiratory physiotherapy in the immediate postoperative period.

In fact, as widely demonstrated and applied in several centers, the Fast-track concept (11) accelerates the patient's recovery after thoracic surgery and decreases the rate of complications.

The chest- $X$ ray was performed after the operation and the day before the expected chest tube removal, that was done when there was no air leak sign and the secretion was below $200-250 \mathrm{~mL}$ within $24 \mathrm{~h}$.

\section{Statistical analysis}

Categorial variables are reported as $\mathrm{n}(\%)$. Continuous variables are expressed as mean \pm standard deviation (SD).

Fischer's exact test was used to compare categorical variables (complications and conversion) and independent sample Student's $t$-test to compare continuous variables among characteristics of the patients between the two groups of U-VATS anatomical resections (lobectomies and segmentectomies).

Any possible correlation between outcome variables (postoperative pain and chest tube duration etc.) was explored by Pearson's sample correlation.

A $P$ value less than 0.05 was considered statistically significant.

Statistical analysis was performed using PASW Statistics for Windows, Version 18.0 (SPSS Inc., Chicago, IL, USA).

\section{Results}

The main demographic and clinical characteristics of the 237 patients underwent U-VATS procedure are reported in Table 1. Among these patients, 5 underwent the operation twice and one three times for iterative lung metastasectomies. The mean age was $59.93 \pm 16.03$ years and there were 121 males (51.1\%) and 116 females (48.9\%).

In 208 cases $(85.3 \%)$ a U-VATS lung resection (Table 2) was performed, in 10 cases $(4.1 \%)$ a U-VATS esophagectomy (only the thorax approach, the creation of a gastric conduit from the stomach and the anastomosis in the neck between the residual esophagus and the newly created gastric tubule were made by mini-laparotomy and cervicotomy) or an esophageal diverticulectomy, in 15 $(6.1 \%)$ a mediastinal procedure and in $11(4.5 \%)$ a toilette for pleural empyema or removal of pleural lesions.

Among the 208 lung resections, $1(0.5 \%)$ was a right pneumonectomy, 43 (20.7\%) lobectomies, 15 (7.2\%) 
Table 2 Type of U-VATS resections and histology

\begin{tabular}{|c|c|}
\hline Resections and histology & $\mathrm{N}=244, \mathrm{n}(\%)$ \\
\hline \multicolumn{2}{|l|}{ Resections } \\
\hline Lung resections & $208(85.3)$ \\
\hline Pneumonectomies & $1(0.5)$ \\
\hline Lobectomies & $43(20.7)$ \\
\hline Segmentectomies & $15(7.2)$ \\
\hline Wedge resections & 149 (71.6) \\
\hline $\begin{array}{l}\text { Esophageal resections (esophagectomy, } \\
\text { diverticulectomy) }\end{array}$ & $10(4.1)$ \\
\hline Mediastinal procedures & $15(6.1)$ \\
\hline $\begin{array}{l}\text { Others (pleural empyema, removal of } \\
\text { pleural lesions) }\end{array}$ & $11(4.5)$ \\
\hline \multicolumn{2}{|l|}{ Histology } \\
\hline NSCLC & $61(25)$ \\
\hline Adenocarcinoma & $50(20.5)$ \\
\hline Squamous cell carcinoma & $6(2.5)$ \\
\hline Others & $5(2.0)$ \\
\hline Lung metastases & $107(43.8)$ \\
\hline Bullous dystrophy & $37(15.2)$ \\
\hline NSIP & $10(4.1)$ \\
\hline $\begin{array}{l}\text { Benign lesions (hamartomas, neuromas, } \\
\text { esophageal diverticula, solitary fibrous } \\
\text { tumors of the pleura, inflammatory } \\
\text { lesions...) }\end{array}$ & $29(11.9)$ \\
\hline
\end{tabular}

NSCLC, non-small cell lung cancer; NSIP, non-specific interstitial pneumonia; U-VATS, uniportal video-assisted thoracic surgery.

segmentectomies and 149 (71.6\%) wedge resections. In $235(96.3 \%)$ cases only one chest tube was inserted, in $9(3.7 \%)$ cases two (manly after pleural debridement for empyema).

The number of U-VATS procedures per month grew enormously together with our expertise, having in the second period of activity $150 \%$ more procedures compared to the first period (150 between February and September 2017 vs. 94 between May 2016 and January 2017, Figure 4).

\section{Operation time and intra-/ perioperative complications}

The mean operation time was $188.21 \pm 53.86 \mathrm{~min}$ for

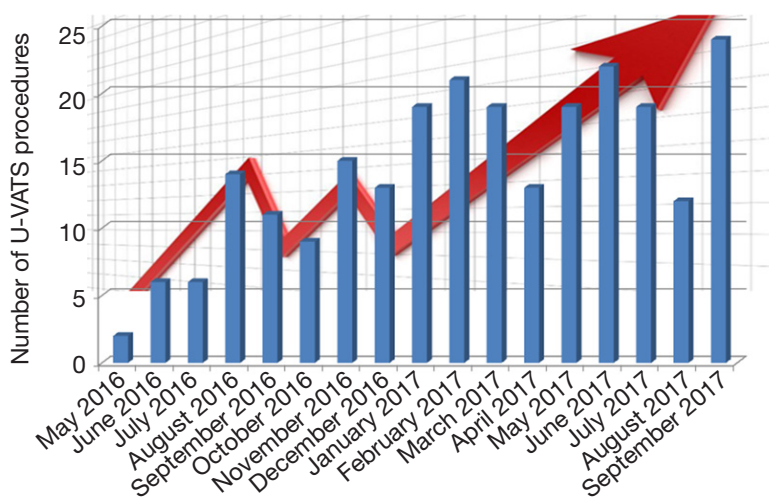

Figure 4 Trend of U-VATS procedures during our 16-month experience. U-VATS, uniportal video-assisted thoracic surgery.

lobectomies, $189.20 \pm 47.60 \mathrm{~min}$ for segmentectomies, $84.57 \pm 38.41 \mathrm{~min}$ for wedge resections, $107.10 \pm 31.05 \mathrm{~min}$ for esophagectomies (only the thorax time of the operation) and $96.07 \pm 34.97 \mathrm{~min}$ for all other procedures.

The intraoperative mortality was null. Five $(2 \%)$ conversions were recorded, because hard adhesions and technical difficulties in 3 cases, arterial injury in 1 case and bronchial injury in another one. The risk for conversion was higher in case of lobectomy compared to other operations $(\mathrm{P}=0.0004)$. And evaluating the main risk factors involved in conversion during lobectomy (complete or incomplete fissure, fissureless technique, adhesions, intraoperative complications...) the presence of hard adhesions turned out to be the only statistically significant factor $(\mathrm{P}<0.001)$.

In one case a second access was needed for better managing a $6 \mathrm{~cm}$ neuroma of the chest wall apex.

There were $2(0.8 \%)$ revisions for postoperative bleeding managed by Uniportal access. Eighteen (7.3\%) patients were admitted to the ICU in the immediate postoperative period only because of their general comorbidities and readmitted to the ward the day after the operation. Three (1.2\%) patients needed noninvasive ventilation in the postoperative period. Among postoperative complications, there were $3(1.2 \%)$ atelectasis, 4 (1.6\%) atrial fibrillations, $3(1.2 \%)$ cases of anemia, $1(0.4 \%)$ bronchopleural fistula, $16(6.5 \%)$ cases of prolonged air-leak $>5$ days and $2(0.8 \%)$ pneumonias.

\section{Histology}

In $168(68.8 \%)$ cases the final pathological finding was a neoplastic lesion, NSCLC in 61 (25\%) cases and lung metastases in 107 (43.8\%). All tumors were completely 
Table 3 Anatomical lung resections: pre-, intra- and postoperative results

\begin{tabular}{|c|c|c|c|}
\hline Variables & Lobectomies (\#43) & Segmentectomies (\#15) & $\mathrm{P}$ \\
\hline $\mathrm{PaCO}_{2}(\mathrm{mmHg})$ & $38.82 \pm 5.25$ & $36.50 \pm 3.64$ & 0.474 \\
\hline preopFEV1\% & $94.00 \pm 17.45$ & $86.50 \pm 0.70$ & 0.566 \\
\hline preopFVC\% & $109.55 \pm 18.83$ & $92.60 \pm 19.83$ & 0.289 \\
\hline Number of lymph nodes removed & $13.40 \pm 9.96$ & $5.92 \pm 3.90$ & $0.015^{*}$ \\
\hline Operation time (min) & $188.21 \pm 53.86$ & $189.20 \pm 47.60$ & 0.968 \\
\hline Conversion & $4(9.3 \%)$ & 0 & 0.215 \\
\hline Complications & $12(27.9 \%)$ & $1(6.7 \%)$ & 0.065 \\
\hline Postoperative stay (days) & $5.57 \pm 2.55$ & $3.53 \pm 1.41$ & $0.005^{*}$ \\
\hline
\end{tabular}

${ }^{*}, \mathrm{P}<0.005$.

resected (R0).

In $76(31.2 \%)$ cases there was no malignancy: 37 (15.2\%) cases were wedge resections for bullous dystrophy for recurrent pneumothorax, 10 (4\%) cases surgical biopsies for confirming a suspected non-specific interstitial pneumonia (NSIP) and in 29 (11.8\%) cases benign lesions of the lung, mediastinum and esophagus (hamartomas, neuromas, esophageal diverticula, solitary fibrous tumors of the pleura, inflammatory lesions...) (Table 2).

\section{Anatomical lung resections}

Lobectomies were performed for the surgical treatment of NSCLC in 38 cases and in 5 for big and central lung metastases (mean dimension of the lesion: $2.77 \pm 1.79 \mathrm{~cm}$ ), while segmentectomies for centimetric primary lung cancers in 11 cases and lung metastases in 4 (Table 3).

Lymphadenectomy was carried out in all NSCLC and the mean number of lymph nodes retrieved was higher after a lobectomy $(13.40 \pm 9.96)$ rather than after a segmentectomy (5.92 $\pm 3.90, \mathrm{P}=0.015)$.

Lobectomy was related to a basically higher incidence of postoperative complications, like persistent air-leakage $>5$ days compared to segmentectomy [8 (18.6\%) patients vs. 0 , respectively, $\mathrm{P}=0.065$ ]. Similarly, a longer chest tube duration was recorded after lobectomy rather than after segmentectomy $(5.90 \pm 4.67$ vs. $3.36 \pm 1.08$ days, $\mathrm{P}=0.049)$.

\section{Short-term results}

In general, the chest tube duration was $4.24 \pm 3.73$ days and the postoperative hospital stay $4.62 \pm 4.59$ days. Thirty-day mortality was null.

At a mean follow-up of $11 \pm 8$ months, among patients operated for a primary lung cancer, only three patients that underwent a lobectomy for large-cell neuroendocrine carcinoma (pathological stage IB or IIA, grading 3), had a recurrence $9.30 \pm 3.60$ months after the operation.

Mean level of pain in I postoperative day was $2.30 \pm 1.26$ on VAS scale (Figure $5 A$ ), the mean duration was of $1.54 \pm 1.21$ days and in $93 \%$ of cases there was a resolution of pain after chest tube removal. The persistence of pain after chest tube removal was unrelated to the chest tube duration $(\mathrm{P}=0.907)$.

For patients who still had pain after drain removal, the level was lower than before $(0.19 \pm 0.57$ on VAS scale), it lasted $1.19 \pm 0.60$ days and was well controlled by intake of nonsteroidal anti- inflammatory drugs or paracetamol. Interestingly, 13 out of 14 patients who underwent previously contralateral thoracotomy confirmed the pain after U-VATS procedure was lower. Patients were also satisfied about cosmetic results (Figure 5B): the average level of satisfaction was $2.73 \pm 0.49$ (measured on a $0-3$ scale). Only 4 (1.6\%) patients developed medium-severe paresthesia 7 days after the operation with spontaneous resolution in about a month. 
A

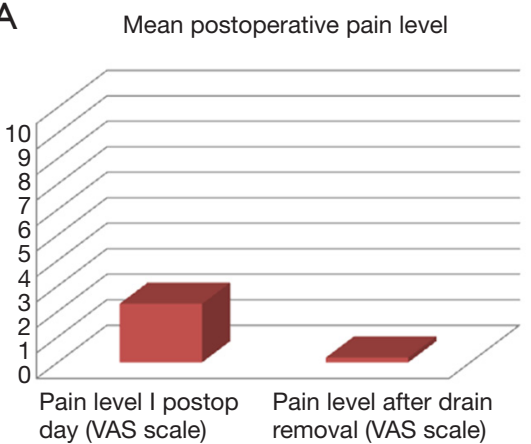

day (VAS scale) removal (VAS scale)
B

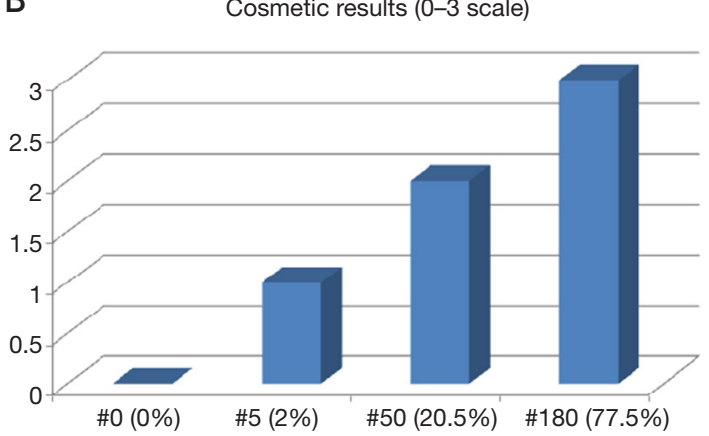

Figure 5 Postoperative pain level (A) and cosmetic results (B).

\section{Discussion}

The development of VATS during the last two decades produced a significant leap in treating a wide range of thoracic diseases. In 1998 Gaetano Rocco (1) was the first pioneer in proposing U-VATS technique and since its introduction U-VATS has been advancing and gaining success among thoracic surgeons.

Nevertheless it was necessary to wait almost two decades to have the first report by Diego Gonzalez-Rivas (2) on a major lung resection with radical lymphadenectomy for non-small cell lung cancer.

Nowadays the U-VATS evolution has been completing worldwide thanks to complex U-VATS performances including segmentectomies, pneumonectomies, bronchoplastic procedures and chest wall resections (3-5) and recently lung resection in non-intubated patient $(6,12-14)$.

Before U-VATS revolution had involved our Hospital, we used to perform major lung resections by Noirclerc's lateral muscle sparing thoracotomy or, in selected cases, by axillary skin incision. Since Nineties we also used single-access VATS for performing pleural biopsies or pleurodesis in order to treat malignant pleural effusion or for sympathectomies in patients affected by primary hyperhidrosis.

In 2013, the availability of the "Da Vinci" robot in our center and our growing interest in mini-invasive thoracic surgery, allowed us to learn and complete the first pulmonary lobectomies and minor lung resections using the robotic technology. In the same year we started a parallel program in triportal VATS for lung resections.

After one year of experience with the triportal VATS, we shifted to biportal VATS because we felt this approach closer to our open antero-lateral approach and used this technique for about 2 years until starting our
U-VATS program in May 2016, after having learned Diego Gonzalez-Rivas's technique (8). According to our experience, U-VATS is more convenient for surgeons, like us, that used to approach pulmonary hilum from the front.

Two stages emphasized the beginning of our training process: the first was the attendance of proper courses and wet-labs and the second was the on-site surgical training with special masterclasses in our Hospital.

Therefore multilevel courses, video libraries, handson conferences and wet-lab experiences are primary and fundamental training opportunities helping in spreading U-VATS worldwide.

Thanks to these courses a thoracic surgeon, with good familiarity with VATS (triportal or biportal), could learn fundamentals and could start to perform U-VATS, easily and safely. Indeed, skilled surgeons, as tutors, guide you in correct use of appropriate instruments for U-VATS, teach you the correct position and movements for camera to avoid fencing and above all help you in creating correct traction on lung parenchyma for each surgical passage and decide the right angulation of the stapler tip, probably two of the hardest steps in U-VATS for beginners.

In fact, it is well established that tutoring programs and training (15) provide an expeditious and effective progression of learning curve facilitating the transition from knowledge to practice.

Therefore, in our 16-month experience we performed 244 cases of U-VATS including major and minor lung resections, esophageal surgery, mediastinal or chest wall procedures, achieving a good learning curve.

At the beginning the unavailability in our center of the special long instruments with proximal and distal articulation was a limiting factor and we had to use conventional endoscopic instruments for a while. In this way 
we experienced that the use of such instruments to perform U-VATS is possible but far from optimal $(9,16)$, resulting in a prolonged operative time but without influencing our learning.

Nowadays U-VATS has become the favorite approach for the treatment of all thoracic pathologies and not only malignancies, at our center.

In this manuscript we are presenting one of the largest Italian series until today. In particular our purpose was to evaluate outcomes in terms of safety and effectiveness of this technique and to analyze short-term results of U-VATS. Moreover, the retrospective analysis of prospectively collected data showed that the U-VATS technique is applicable for both major and minor pulmonary resections, thoracic esophageal diseases and, finally, it represents a valid option for surgical approach to different pathologies of the mediastinum, pleura and thoracic wall.

Among the main advantages of this technique, we observed the reduction of post-operative hospital stay, above all for wedge resections and segmental resections $(3.53 \pm 1.41$ days). Although in lobectomy group the average hospital stay was about 5 days, it was lower than that of open lung lobectomies in our hospital. In general, our results in terms of hospital stay after a U-VATS procedure were in line with those of other series reported in literature (17-19). The reduction of post-operative hospitalization was possible thanks to factors such as the early removing of pleural drainage and low incidence of postoperative complications, like pneumonias, atelectasis and respiratory or cardiac problems.

Furthermore, we documented a marked reduction of post-operative pain, and the consequent use of analgesics. Finally, the cosmetic results of the surgical incision were evaluated. The $3-4 \mathrm{~cm}$ incision and the intradermal sutures performed with absorbable thread allowed an excellent final result. All this factors allowed a better quality of life and a faster post-surgical recovery for patients.

The main limitation of this study was the lack of oncologic outcomes because of the short follow-up. In fact, in line with literature, it has not been possible to completely express it in terms of global survival and diseasefree survival, yet. To date there are no results about any randomized controlled prospective studies comparing U-VATS to conventional VATS or to open thoracotomy in terms of oncologic outcomes. On the other hand, there are several recent retrospective publications comparing U-VATS to traditional VATS or to open surgery that showed advantages of U-VATS technique about non- oncologic outcomes (20,21).

Stating this, our experience suggested that U-VATS is a safe and practicable mini-invasive technique for all surgeons who already have thoracoscopic experience. Furthermore we report some other reasons why the U-VATS approach might be preferred: the approach to the target lesion is similar to open surgery, the involvement of only one intercostal space reduces intercostal pain considerably and gives a cosmetically advantage and, mainly, this technique allows a brief hospital stay and lower incidence of complications, realizing the goal of fast-track surgery. Finally, the incessant improvement in U-VATS dedicated instrumentation (devices for hemostasis and dissection energy or articulated staplers) are facilitating surgeons learning curve and performance in this technique testifying that the U-VATS revolution has been advancing continuously and it is far from ending.

\section{Acknowledgements}

None.

\section{Footnote}

Conflicts of Interest: The authors have no conflicts of interest to declare.

Ethical Statement: This study was evaluated by the Institutional Review Board (IRB) of Catholic University of Sacred Hearth and, as this was a retrospective review for service evaluation (within an audit approved by our Surgical Department) and there was no modification in patients' care (no prospective randomized study), we did not need the final ethical approval of our IRB. All patients provided written informed consent before the surgical operation for the treatment of their clinical data.

\section{References}

1. Rocco G, Martin-Ucar A, Passera E. Uniportal VATS wedge pulmonary resections. Ann Thorac Surg 2004;77:726-8.

2. Gonzalez-Rivas D, de la Torre M, Fernandez R, et al. Single-port video-assisted thoracoscopic left upper lobectomy. Interact Cardiovasc Thorac Surg 2011;13:539-41.

3. Gonzalez-Rivas D. VATS lobectomy: surgical evolution from conventional VATS to uniportal approach. 
ScientificWorldJournal 2012;2012:780842.

4. Gonzalez-Rivas D, Mendez L, Delgado M, et al. Uniportal video-assisted thoracoscopic anatomic segmentectomy. J Thorac Dis 2013;5 Suppl 3:S226-33.

5. Gonzalez-Rivas D, Delgado M, Fieira E, et al. Double sleeve uniportal video-assisted thoracoscopic lobectomy for non-small cell lung cancer. Ann Cardiothorac Surg 2014;3:E2.

6. Gonzalez-Rivas D. Uniportal thoracoscopic surgery: from medical thoracoscopy to non-intubated uniportal videoassisted major pulmonary resections. Ann Cardiothorac Surg 2016;5:85-91.

7. Eckland K, Gonzalez-Rivas D. Teaching uniportal VATS in Coruña. J Vis Surg 2016;2:42.

8. Meacci E, Nachira D, Congedo MT, et al. Teaching uniportal video-assisted thoracic surgery in Rome. J Vis Surg 2017;3:49.

9. Ng CS. Uniportal VATS in Asia. J Thorac Dis 2013;5 Suppl 3:S221-5.

10. Ismail M, Swierzy M, Nachira D, et al. Uniportal videoassisted thoracic surgery for major lung resections: pitfalls, tips and tricks. J Thorac Dis 2017;9:885-97.

11. Ismail M, Swierzy M, Nachira D, et al. Fast-Tracking Patients Through the Diagnostic and Therapeutic Pathways of Intrathoracic Conditions: The Role of Uniportal Video-Assisted Thoracic Surgery. Thorac Surg Clin 2017;27:425-30.

12. Rocco G. Non-intubated uniportal lung surgery†. Eur J

Cite this article as: Nachira D, Meacci E, Petracca Ciavarella L, Chiappetta M, De Santis G, Ferretti GM, Mastromarino MG, Porziella V, Vita ML, Congedo MT, Cesario A, Ismail M, Gonzalez-Rivas D, Margaritora S. Uniportal video-assisted thoracic surgery Roman experience-a report of the first 16-month Roman experience. J Thorac Dis 2018;10(Suppl 31):S3678-S3685. doi: 10.21037/jtd.2018.03.119
Cardiothorac Surg 2016;49 Suppl 1:i3-5.

13. Zheng $\mathrm{H}, \mathrm{Hu} \mathrm{XF}$, Jiang GN, et al. Nonintubated-Awake Anesthesia for Uniportal Video-Assisted Thoracic Surgery Procedures. Thorac Surg Clin 2017;27:399-406.

14. Li S, Jiang L, Ang KL, et al. New tubeless video-assisted thoracoscopic surgery for small pulmonary nodules. Eur J Cardiothorac Surg 2017;51:689-93.

15. Kwon EO, Bautista TC, Blumberg JM, et al. Rapid implementation of a robot-assisted prostatectomy program in a large health maintenance organization setting. J Endourol 2010;24:461-5.

16. Gonzalez-Rivas D, Paradela M, Fernandez R, et al. Uniportal video-assisted thoracoscopic lobectomy: two years of experience. Ann Thorac Surg 2013;95:426-32.

17. Ismail M, Helmig M, Swierzy M, et al. Uniportal VATS: the first German experience. J Thorac Dis 2014;6:S650-5.

18. Abu Akar F, Gonzalez-Rivas D, Ismail M, et al. Uniportal video-assisted thoracic surgery: the Middle East experience. J Thorac Dis 2017;9:871-7.

19. Bondulich G, Gonzalez Rivas D. Uniportal video-assisted thoracoscopic surgery, Argentinian experience. J Vis Surg 2017;3:60.

20. Hirai K, Enomoto Y, Usuda J. For which thoracic operation is U-VATS superior? J Vis Surg 2017;3:103.

21. Guerrero WG, González-Rivas D. Multiportal videoassisted thoracic surgery, uniportal video-assisted thoracic surgery and minimally invasive open chest surgeryselection criteria. J Vis Surg 2017;3:56. 